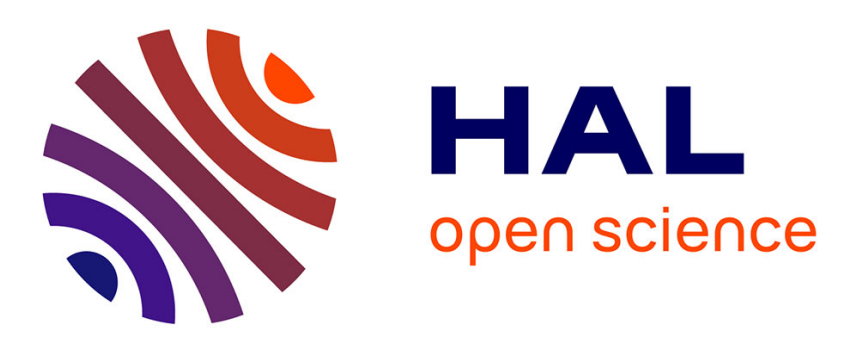

\title{
Steering capabilities of an acoustic lens for transcranial therapy: numerical and experimental studies
}

\author{
Guillaume Maimbourg, Alexandre Houdouin, Thomas Deffieux, Mickaël \\ Tanter, Jean-François Aubry
}

\section{- To cite this version:}

Guillaume Maimbourg, Alexandre Houdouin, Thomas Deffieux, Mickaël Tanter, Jean-François Aubry. Steering capabilities of an acoustic lens for transcranial therapy: numerical and experimental studies. IEEE Transactions on Biomedical Engineering, 2018, pp.1-1. 10.1109/TBME.2019.2907556 . hal02411218

\author{
HAL Id: hal-02411218 \\ https://hal.science/hal-02411218
}

Submitted on 14 Dec 2019

HAL is a multi-disciplinary open access archive for the deposit and dissemination of scientific research documents, whether they are published or not. The documents may come from teaching and research institutions in France or abroad, or from public or private research centers.
L'archive ouverte pluridisciplinaire HAL, est destinée au dépôt et à la diffusion de documents scientifiques de niveau recherche, publiés ou non, émanant des établissements d'enseignement et de recherche français ou étrangers, des laboratoires publics ou privés. 


\title{
Steering capabilities of an acoustic lens for transcranial therapy: numerical and experimental studies
}

\author{
Guillaume Maimbourg $^{1,2,3,4}$, Alexandre Houdouin ${ }^{1,2,3}$, Thomas Deffieux ${ }^{1,2,3}$, Mickael Tanter ${ }^{1,2,3}$ and Jean-François Aubry ${ }^{1,2,3}$ \\ 1- INSERM U979, Institut Langevin, Paris, France; \\ 2- ESPCI Paris, PSL Research University, Institut Langevin, Paris, France; \\ 3- CNRS UMR 7587, Institut Langevin, Paris, France; \\ 4- Université Paris Diderot, Paris, France
}

\begin{abstract}
Brain therapy by transcranial focused ultrasound needs to compensate for the delays locally induced by the skull. The patient-specific phase profile is currently generated by a multi-element array with a growing number of elements. We recently introduced a disruptive approach, consisting in using a single element transducer coupled to an acoustic lens of controlled thickness: by adjusting the local thickness of the lens, we were able to induce a phase difference which compensated that of the skull. Nevertheless, such an approach suffers from an apparent limitation: the lens is a priori designed for one specific target. In this paper, we demonstrate the possibility of taking advantage of the isoplanatic angle of the aberrating skull in order to steer the focus by mechanically moving the transducer/acoustic lens pair around its initial focusing position. This study, conducted on three human skull samples, confirms that tilting of the transducer with the lens restores a single focus at $914 \mathrm{kHz}$ for a steering up to $11 \mathrm{~mm}$ in the transverse direction, and $10 \mathrm{~mm}$ in the longitudinal direction, around the initial focal region.
\end{abstract}

Acoustic lens, transcranial ultrasound, therapeutic ultrasound

\section{INTRODUCTION}

Focused ultrasound therapies have seen clinical applications for a growing number of pathologies: prostate $[1,2]$ or pancreatic $[3,4]$ cancers, bone metastases $[5,6]$, uterine fibroids [7-9], or essential tremor [10-12]. It complements or even advantageously replaces surgery-based conventional methods. One of the prime advantages of the method is that it is non-invasive or minimally-invasive, and thus reduces convalescence time and post-operative risks of complications. It has however remained inapplicable to the human brain for a long time, due to the defocusing effect of the skull $[13,14]$. In the 90s, an innovative technique, based on wavefront control and time reversal was proposed [15-18], before being tested on animals [19-21] and cadavers [22-24]. Transcranial focusing can be used with a diverse range of modi operandi, including Blood-Brain-Barrier (BBB) opening [25-29], thermal lesioning $[20,21,30]$, as well as others, like

Submitted on 2018, May $1^{\text {st }}$

This work was supported by the Bettencourt Schueller Foundation and the

"Agence Nationale de la Recherche" under the program "Future Investments" with the reference ANR-10-EQPX-15. neuromodulation [31-37]- some of which are already being used in clinics [10, 38-40].

The phase-corrected profile is usually generated by a multielement array: each element emits a signal with a precomputed phase so as to compensate for the delays locally induced by the skull. The quality of the focusing being directly linked to the number of elements, the progress made in electronics and transducer conception led to the development of probes featuring an increasing number of elements: 64 elements in 2000 [41], 200 in 2003 [42], 512 in 2013 [22], 1024 in 2010 [43] or 1372 also in 2010 [44]. Inspired by previous studies employing acoustic lenses for therapeutic ultrasound [45-52], we introduced a disruptive approach[53], consisting in using a single element transducer coupled to an acoustic lens of controlled thickness: by adjusting the local thickness of the lens, we were able to induce phase differences which compensated that of the skull. With such acoustic lenses, we were able to focus through a set of 3 human skulls, and show comparable levels of precision and efficiency to those attained by conventional, multi-element systems. Nevertheless, such an approach suffers from an inherent limitation: each lens is designed for one given patient and one given target[54], meaning that - for a given patient - a surgeon could neither change the target after the fabrication of the lens, nor treat a large area over the course of the surgery, without making a new lens. Correcting aberrations with an acoustic lens thus seemed like a less flexible method than using multi-element arrays, with which new phase sets can be electronically changed to aim at another target during treatment procedure[42]. In this paper, we propose to take advantage of the isoplanatic angle of the aberrating skull in order to steer the focus by mechanically moving the transducer/acoustic lens pair around its initial focusing position, similarly to what is done in astronomy to compensate the aberrations induced by the atmosphere[55]. This study was conducted on three human skulls, first numerically, and then experimentally. The quality of the focusing is assessed for a longitudinal and transverse translation of the focus.

\section{MATERIALS AND METHODS}

\section{A. Transducer}


A single-element transducer with a $61 \mathrm{~mm}$ radius of curvature and a $67 \mathrm{~mm}$ aperture (H101 MR, Sonic Concepts, Bothell, USA) was used to achieve transcranial focusing. It was operated at its resonance frequency $(914 \mathrm{kHz})$, which was determined from the analysis of its complex impedance against frequency, using a vector network analyzer (ZVL, Rohde \& Schwarz, Munich, Germany). Prior to the study, we evaluated that this transducer had a $91 \%$ electro-acoustic conversion efficiency at its resonance frequency.

\section{B. Human skull}

The experiments were conducted on $\mathrm{N}=3$ human skulls (hereafter referenced as A, B and C). The human skulls were provided by the Institut d'Anatomie (UFR Biomédicale des Saints-Pères, Université Paris Descartes, Paris, France) and tattooed with individual numbers, as approved by the ethics committee of the Centre du Don des Corps (Université Paris Descartes, Paris, France). The skulls were immersed in water and degassed under a $2 \mathrm{mbar}$ reduced pressure (diaphragm

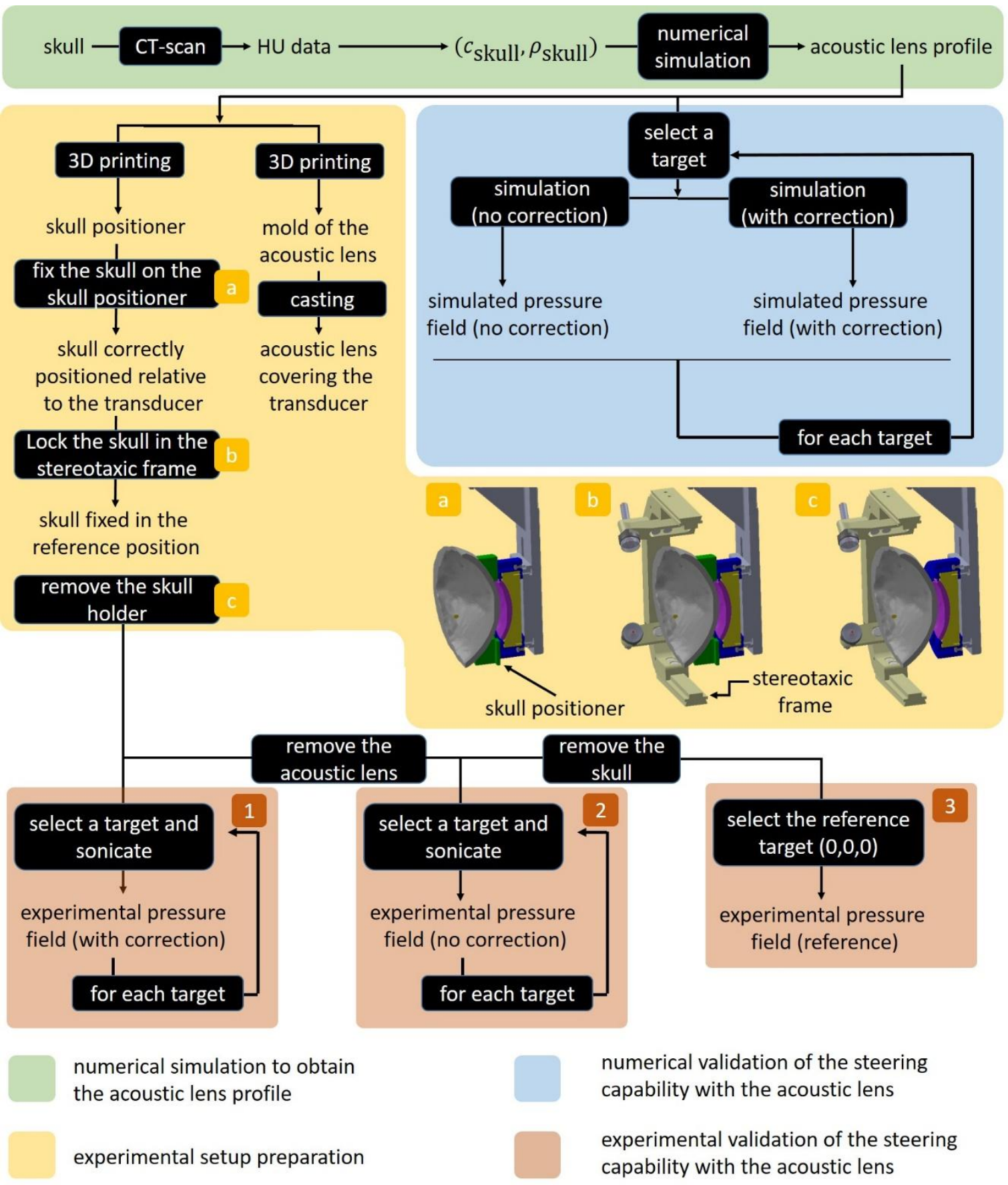

Figure 1: Synoptic view of the whole numerical and experimental protocols. The four major phases are colored for easier identification.

pump FB65457, Fischer Scientific, Waltham, MA, USA) during 48h; their CT-scans were acquired (Sensation 64, 
Siemens, Erlangen, Germany) at the CHU de Marseille Nord, France. The in-plane spatial resolution of the slices was $0.41 \mathrm{~mm}$, while slice thickness and interslice spacing were $0.60 \mathrm{~mm}$ and $0.30 \mathrm{~mm}$, respectively. The speed of sound and density maps of the skull used in the numerical simulations were derived by a linear approximation from these HU data[56].

\section{Determining the thickness of the acoustic lens through numerical simulation}

In order to determine the thickness of the corrective acoustic lens, an acoustic wave was emitted from the target point (Figure 1, green box) and numerically propagated across the skull. The simulated emitter was placed at a known location relative to the skull, which enabled us to target a predefined region. A 3-dimension linear interpolation of the speed of sound and density maps was performed to obtain a $\lambda_{\text {water }} / 10$ spatial step in each dimension. The time step $\Delta t_{\text {sim }}$ was computed based on a 0.3 Courant-Friedrichs-Lewy (CFL) condition in the cortical bone. The simulation was conducted using a k-space pseudospectral method-based solver, k-Wave [57]. Neither the absorption properties of the skull nor those of the tissues were taken into account. To further reduce the simulation time, the point source at the target was replaced with an equivalent spherical wavefront originated from the target and adjusted as close as possible to the brain-skull interface. Erreur! Source du renvoi introuvable.Figure 4(left) shows a cross-section view of the numerical simulation setup. A 2-cycle sinusoidal burst with Gaussian apodization was emitted at the frequency $f_{0}=914$ $\mathrm{kHz}$ and numerically propagated in the medium. The signal was recorded on the surface of the transducer and the phase $\varphi$ of the signal was evaluated by a projection onto the Fourier basis. The lens thickness was then 2D-unwrapped on the surface of the transducer to avoid phase discontinuities. The local thickness $p$ of the lens on every point $M$ of the transducer is evaluated, according to the following formula, and where $\tilde{\varphi}$ is the phase after unwrapping:

$$
p(M)=\frac{\widetilde{\varphi}(\mathrm{M})}{2 \pi f_{0}} \cdot \frac{1}{1 / c_{\text {water }}-1 / c_{\text {lens }}}+K
$$

$K$ is a constant length chosen to set the minimal thickness of the lens. In our study, $K$ was set to $2 \mathrm{~mm}$. The speed of sound was $c_{\text {water }}=1485 \mathrm{~m} / \mathrm{s}$ [58] and $c_{\text {lens }}=1000 \mathrm{~m} / \mathrm{s}$ [53] respectively in water and in the acoustic lens.

The simulation was run on a workstation equipped with two 2.20GHz Intel Xeon CPU E5-2630v4 processors and 32GB RAM; the full running time was about one hour.

More details about the method used to build the acoustic lens can be found in Maimbourg et al.[53].

\section{Mechanism of the experimental setup}

A mold was 3D-printed (Replicator 2X, Makerbot, Brooklyn, NY, USA) based on the shape that had been determined from the numerical simulation (Figure 1, yellow box). The acoustic lens was then cast with a commercial silicone (Elite double 8, Zhermack, Badina Polesine, Italy). A plastic holder of shape complementary to the skull was also 3D-printed, in order to position the transducer relative to the skull in a low lost and effective manner. The positioning of the skull was achieved by a three-step process summarized in Figure 1, yellow box:

(a) The skull is positioned relatively to the transducer thanks to the skull positioner.

(b) The screws of the stereotaxic frame are tightened so that the skull remains still.

(c) The transducer is moved backwards, and the skull positioner removed.

At the end of these steps, the skull was in a known position relatively to the transducer, all the while allowing for the movement of the transducer relatively to the skull, which allowed us to test mechanical steering. Figure 2.A indicates the geometrical coordinate system chosen for the study. The transducer was now mounted onto a linear stage (UE71PP, Micro-Controle, Newport, CA, USA) on the $\mathrm{z}$ axis and a rotary stage (TR80-BL, Micro-Controle, Newport, CA, USA) on the (Cy) axis, where $\mathrm{C}$ is the point corresponding to the center of the surface of the transducer. Using this setup, the precision was $0.01 \mathrm{~mm}$ in linear A-P displacement, and 1' in rotation. These two degrees of freedom allowed for steering along the $x$ (antero posterior axis of the skull) and $Z$ (longitudinal axis of the skull) axes. Figure 3, as well as the corresponding table, recapitulate the targeted positions, along with the $\theta_{y}$ and $T_{z}$ rotations required to reach them. To obtain an $x$-axis translation, we combined a $\theta_{y}$ rotation and a $T_{z}$ translation (Figure 2.B). With $f=61 \mathrm{~mm}$ being the focal distance of the transducer, the following formulae link $\theta_{y}$ and $T_{z}$ to the desired $x$ translation:

$$
\theta_{y}=\sin ^{-1}\left(\frac{x}{f}\right) \quad \text { and } \quad T_{z}=f-\sqrt{f^{2}-x^{2}}
$$

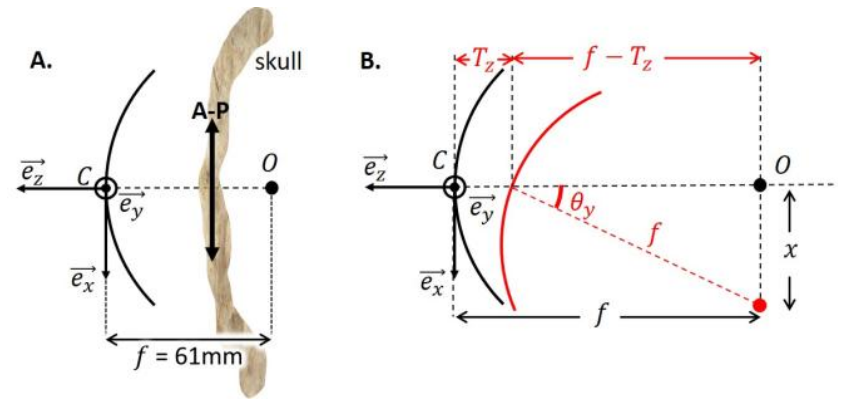

Figure 2: A. Coordinate system used in the study. Its origin $O$ corresponds to the target, the $x$ direction to the Antero-Posterior (A$P)$ axis of the skull. B. Evidencing the $z$ and $\theta_{y}$ movements required to steer toward the $x$ direction.

(2)

Due to the proximity between the acoustic lens and the skull, every steering configuration was not feasible. Indeed, for certain configurations, the acoustic lens interfered with the skull. It was not possible to attempt steering in the $+\overrightarrow{e_{z}}$ direction in particular, and skull $\mathrm{C}$ was the only one that could allow testing for positive and negative steering along $x$. We assumed that steering positions with positive and negative $x$ were equivalent for the purpose of the study. As a consequence, the positive and negative steerings for skull $\mathrm{C}$ were concatenated in the Results section. The table on 3 shows 
the tested configurations for each skull. During a preliminary

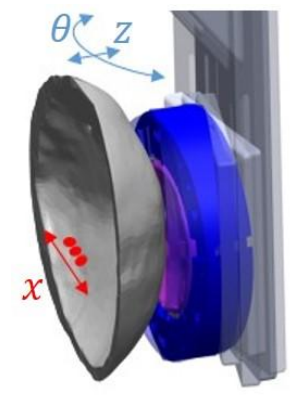

\begin{tabular}{lcccccc}
\hline \hline & $\begin{array}{c}\text { X-steering } \\
(\mathrm{mm})\end{array}$ & $\begin{array}{c}\text { Z-stage } \\
(\mathrm{mm})\end{array}$ & $\theta_{y^{-} \text {-stage }}$ & \multicolumn{3}{c}{ SKULL } \\
\hline$x_{5}{ }^{\prime}{ }^{\prime}$ & -11.2 & -1.0 & $10^{\circ} 35^{\prime}$ & & $\bullet$ & $\mathrm{C}$ \\
$x_{4}{ }^{\prime}$ & -9.0 & -0.7 & $8^{\circ} 28^{\prime}$ & & $\bullet$ & \\
$x_{3}{ }^{\prime}$ & -6.8 & -0.4 & $6^{\circ} 21^{\prime}$ & & $\bullet$ & \\
$x_{2}{ }^{\prime}$ & -4.5 & -0.2 & $4^{\circ} 14^{\prime}$ & & $\bullet$ & \\
$x_{1}{ }^{\prime}$ & -2.3 & 0.0 & $2^{\circ} 7^{\prime}$ & & $\bullet$ & \\
$x_{0}$ & 0.0 & 0.0 & $0^{\circ}$ & $\bullet$ & $\bullet$ & $\bullet$ \\
$x_{1}$ & 2.3 & 0.0 & $2^{\circ} 7^{\prime}$ & $\bullet$ & $\bullet$ & $\bullet$ \\
$x_{2}$ & 4.5 & 0.2 & $4^{\circ} 14^{\prime}$ & $\bullet$ & $\bullet$ & $\bullet$ \\
$x_{3}$ & 6.8 & 0.4 & $6^{\circ} 21^{\prime}$ & $\bullet$ & $\bullet$ & $\bullet$ \\
$x_{4}$ & 9.0 & 0.7 & $8^{\circ} 28^{\prime}$ & $\bullet$ & $\bullet$ & $\bullet$ \\
$x_{5}$ & 11.2 & 1.0 & $10^{\circ} 35$, & $\bullet$ & $\bullet$ & $\bullet$ \\
\hline \hline
\end{tabular}

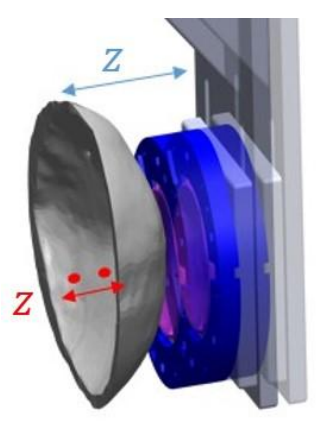

\begin{tabular}{ccccccc}
\hline \hline & $\begin{array}{c}\text { z-steering } \\
(\mathrm{mm})\end{array}$ & $\begin{array}{c}\text { Z-stage } \\
(\mathrm{mm})\end{array}$ & $\theta_{y^{-} \text {-stage }}$ & \multicolumn{3}{c}{ SKULL } \\
\hline$x_{0}$ & 0.0 & 0.0 & $0^{\circ}$ & $\bullet$ & $\bullet$ & $\bullet$ \\
$x_{1}$ & 2.0 & 2.0 & $0^{\circ}$ & $\bullet$ & $\bullet$ & $\bullet$ \\
$x_{2}$ & 4.0 & 4.0 & $0^{\circ}$ & $\bullet$ & $\bullet$ & $\bullet$ \\
$x_{3}$ & 6.0 & 6.0 & $0^{\circ}$ & $\bullet$ & $\bullet$ & $\bullet$ \\
$x_{4}$ & 8.0 & 8.0 & $0^{\circ}$ & $\bullet$ & $\bullet$ & $\bullet$ \\
$x_{5}$ & 10.0 & 10.0 & $0^{\circ}$ & $\bullet$ & $\bullet$ & $\bullet$ \\
\hline \hline
\end{tabular}

Figure 4: Longitudinal and transverse steering configurations. The values of the $z$ translation and of the $\theta_{y}$ rotation are provided for each.

study, we used a first transducer with a different focal depth; for such a focus, the $\theta_{y}$ angle should be incremented by $2^{\circ} 7^{\prime}$ steps to obtain $2 \mathrm{~mm}$ displacements along $x$. For practical reasons, we kept this angular step throughout the rest of our study, resulting in the $x$-steering step being a non-round value (for $\theta_{y}=2^{\circ} 7^{\prime}$, the $x$-steering equals $2.3 \mathrm{~mm}$ ).

\section{E. Numerical assessment of the steering capability}

We first assessed numerically the capabilities of the method (Figure 1, blue box). To this end, a sinusoidal wave was emitted at $914 \mathrm{kHz}$ from the surface of the transducer and propagated through the acoustic lens and the skull (Figure 4, right). The numerical simulation was conducted according to the same specifications (speed of sound and density maps, spatial step, CFL conditions) that the ones used when determining the thickness of the lens. It was, however, conducted on a larger volume that included the target (white dashed box, on Figure 2, right). The pressure field was recorded on a $x \times y \times z=8 \times 8 \times 16 \mathrm{~mm}^{3}$ volume around the target. In order to simulate focusing at different positions, the speed of sound and density maps representing the skull were rotated and displaced while the transducer and the lens remained static. All the positions that were experimentally tested (Figure 3 ) were tested numerically first.

\section{F. Experimental assessment of the steering capability}

The pressure field was recorded for each steering position (Figure 1, red boxes). An arbitrary waveform generator (AFG3101C, Tektronix, Beaverton, OR, USA) emitted a 200cycles sinusoidal burst at $914 \mathrm{kHz}$. This signal was then amplified (40AD1, Amplifier Research, Souderton, PA, USA). An oscilloscope (DPO3034, Tektronix, Beaverton, OR, USA) recorded the input and output signals of the amplifier. The acoustic pressure field was measured with a needle hydrophone (HNA-0400, Onda Corp., Sunnyvale, CA, USA) mounted on a 3-axis positioning system (ESP301 and ILS Linear Stages, Newport, CA, USA). The $\mathrm{x}, \mathrm{y}$ and $\mathrm{z}$ axes corresponded to the linear stages, so that $\mathrm{z}$ corresponded to the longitudinal axis of the transducer (Figure 2.A). The output signal of the hydrophone was amplified (AG-2020 and AH2020-DCBSW, Onda Corp., Sunnyvale, CA, USA) before digitalization at the sampling frequency $f_{s}=100 \mathrm{MHz}$ on $N=1$ MPts using a data acquisition card (HS5, TiePie, Sneek, Netherlands). Prior to the experiments, the hydrophone was calibrated according to an absolute measure of pressure, using

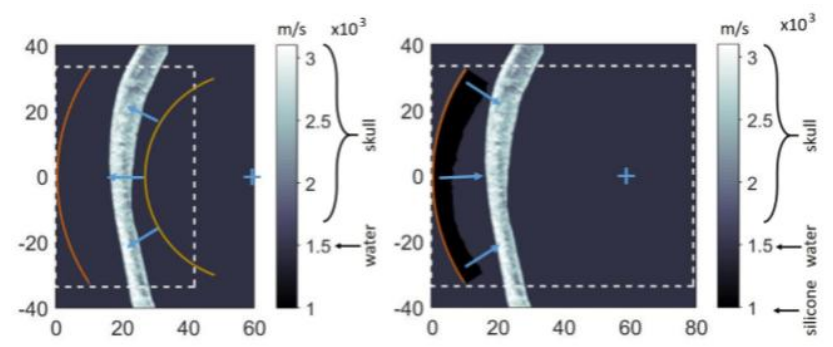

Figure 3: Left: Speed of sound map of skull A in a cross-section plane defined by $x=0$. The blue cross represents the target and coincides with the geometrical focus of the transducer. The yellow arc centered on the target is the source used in the simulation. The red surface represents the surface of the transducer where the acoustic signal is recorded, after propagation through the skull. The white dash box delimits the area in which the simulation was calculated. Right: The wave is emitted from the surface of the transducer and propagates through the acoustic lens and the skull. The pressure field is acquired in a volume centered on the target. 
a heterodyne interferometer [59]. A 1.40V/MPa sensitivity was obtained. All the devices were synchronized using a computer, thanks to an in-house Matlab code (Mathworks, Natick, MA, USA). For each position of the hydrophone, the signal was averaged throughout 5 acquisitions. In order to extract the harmonic component from the acoustic signal, the average signal was projected onto the Fourier basis corresponding to the excitation frequency (914 $\mathrm{kHz}$ ). As illustrated in Figure 1, red boxes, the pressure field was recorded in three cases: (1) propagation through the skull with the acoustic lens correction, (2) propagation through the skull (3) propagation through water only (only recorded for the target $(0,0,0))$. The pressure field obtained through water only served as a reference for the target before steering $(0,0,0)$. Then, for example, the target for a $2 \mathrm{~mm} \mathrm{x}$-steering was assumed to be located at $(2,0,0)$.

\section{G. Data analysis}

The acoustic intensity $I_{\mathrm{ac}}$ was evaluated for each case, using the pressure fields obtained both experimentally and by numerical simulation:

$$
I_{\mathrm{ac}}=\frac{A_{m}^{2}}{2 Z}
$$

where $A_{m}$ is the pressure amplitude and $Z$ the impedance of water.

During the experiments, the acoustic intensity obtained for the acoustic lens correction was corrected from the attenuation induced by the lens, as explained in [53]. This allows direct comparison with the simulations, in which the attenuation of the lens is not modeled. Comparisons were thus carried out with equal intensity on the outer surface of the skull.

The position of the acoustic focus was estimated by calculating the barycentric coordinates of the intensity within the $-6 \mathrm{~dB}$ main focal region.

(4)

$$
\overrightarrow{O F}=\left(\begin{array}{l}
x_{F} \\
y_{F} \\
z_{F}
\end{array}\right)=\frac{\sum_{\mathrm{V} @-6 \mathrm{~dB}} I_{\mathrm{ac}}(M) \overrightarrow{O M} \Delta x \Delta y \Delta z}{\sum_{\mathrm{V} @-6 \mathrm{~dB}} I_{\mathrm{ac}}(M) \Delta x \Delta y \Delta z}
$$

The longitudinal error $\varepsilon_{\text {longi }}$ and transverse error $\varepsilon_{\text {trans }}$ were then calculated:

$\varepsilon_{\text {trans }}=\sqrt{\left(x_{F}-x_{T}\right)^{2}+\left(y_{F}-y_{T}\right)^{2}}$ and $\varepsilon_{\text {longi }}=\left|z_{F}-z_{T}\right|$

where $\left(x_{T}, y_{T}, z_{T}\right)$ is the coordinate of the target.

The surface of the $-3 \mathrm{~dB}$ focal spot on the transverse plane was finally calculated, in order to assess the dispersion of the acoustic power.

All these metrics were evaluated for skulls A, B and C, with and without the correction provided by the lens, for both experimental and numerical results.

\section{RESULTS}

\section{A. Numerical and experimental evaluations of steering capabilities along the transverse direction $x$}

Figure 5 shows the $-3 \mathrm{~dB}$ focus area for a transducer mechanically steered along the $\mathrm{x}$ axis. The results for the three
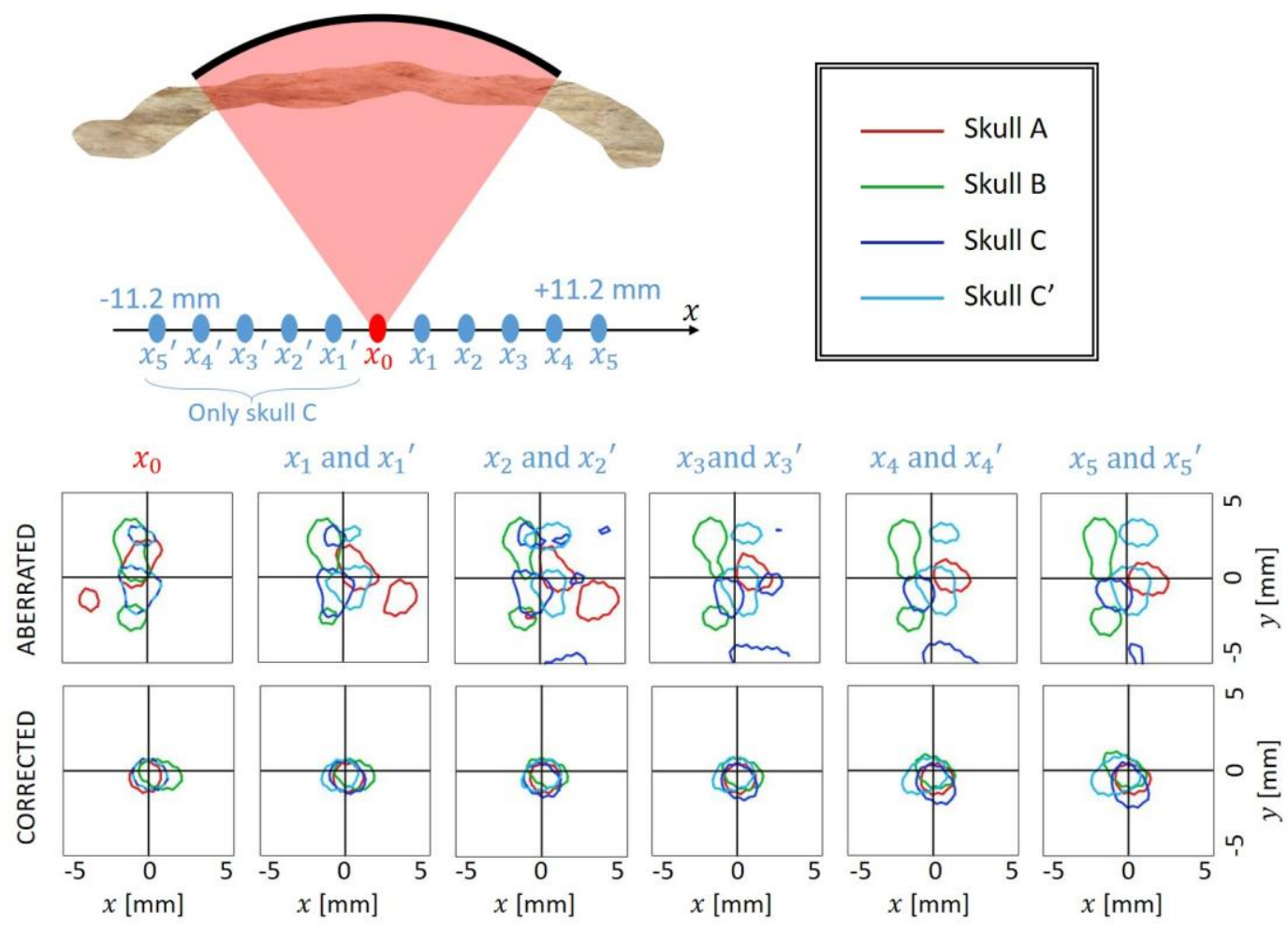

Figure 5: $-3 d B$ focus area in the transverse plane for a transducer mechanically steered along the $x$ axis. The results for the three skulls (and for both positive and negative steering, in the case of skull $C$ ) are presented on top of one another. The top row displays the results obtained experimentally for a case where the wave propagated across a skull without the correction of the acoustic lens. The bottom row displays the results obtained after correction by the acoustic lens. The black cursor represents the desired target. 

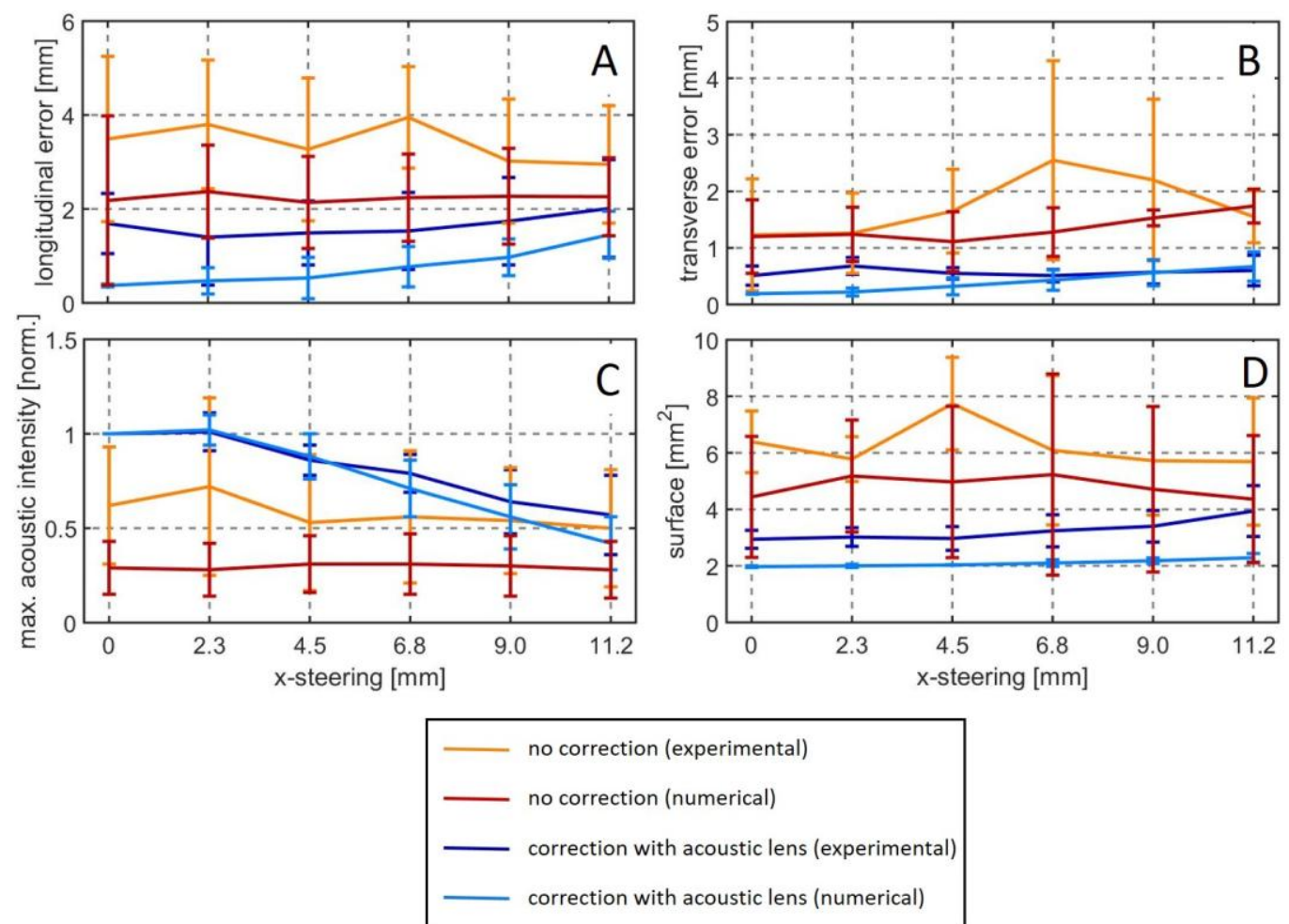

Figure 6: A) Average and standard deviation of longitudinal error $\varepsilon_{\text {longi }}$ (equation 5), as a function of steering along the $x$ axis. B) Average and standard deviation of transverse error $\varepsilon_{\text {trans }}$ (equation 5), as a function of steering along the $x$ axis. C) Average and standard deviation of maximum acoustic intensity (normalized by the acoustic intensity obtained with the acoustic lens correction without steering), as a function of steering along the $x$ axis. D) Average and standard deviation of the surface of the $-3 d B$ focal spot in the transverse plane, as a function of steering along the $x$ axis.

skulls (and for both positive and negative steering, in the case of skull C) are presented on top of one another. The top row displays the results obtained experimentally without the acoustic lens correction. Without any correction, multiple foci are always present and focusing quality is poor. More energy is dispersed along the $y$ axis (the transverse axis of the skull) than along the $\mathrm{x}$ axis, in the majority of cases shown here. The dispersion tends to moderately increase as well, as the steering angle increases. The bottom row displays the results obtained with the acoustic lens correction. A unique focus was obtained in every case. The dispersion of acoustic energy does not noticeably increase when the acoustic lens is added, and said dispersion does not noticeably increase either for $\mathrm{x}$-direction steering, up to $11.2 \mathrm{~mm}$ (position $\mathrm{x}_{5}$ and $\mathrm{x}_{5}{ }^{\prime}$ ). The precision of the focusing suffers from greater steering, however.

Figure 6 quantitatively summarizes these results and shows the longitudinal and transverse errors, the maximum acoustic intensity, as well as the surface of the $-3 \mathrm{~dB}$ area in the $\mathrm{XY}$ (transverse) plane. Averages and standard deviations are calculated for all three skulls, with and without correction by the lens, for both numerical and experimental results. Without correction, the curves do not follow a clear trend relatively to an increase in steering along the $\mathrm{x}$ axis. With the correction provided by the acoustic lens, the longitudinal and transverse errors are lower than $2 \mathrm{~mm}$ and $1 \mathrm{~mm}$ respectively for every steering position. As far as the maximum acoustic intensity is concerned, its level drops to about a half $(43 \pm 21 \%$ and
$68 \pm 14 \%$ respectively experimentally and numerically) for an $11.2 \mathrm{~mm}$ steering, compared with the reference target without steering. The $-3 \mathrm{~dB}$ surface in the $\mathrm{XY}$ plane is about twice as narrow with correction - and increases with steering. Compared to the reference, it grows by $34 \pm 42 \%$ experimentally and by $16 \pm 9 \%$ numerically for an $11.2 \mathrm{~mm}$ steering along the $\mathrm{x}$ axis.

\section{B. Numerical and experimental evaluations of the steering capabilities along the longitudinal direction $z$}

Figure 7 shows the $-3 \mathrm{~dB}$ focus area when the transducer is mechanically steered along the $\mathrm{z}$ axis. The results for the three skulls are drawn on top of one another. The top row represents experimental results through the skull without the acoustic lens correction. Without correction, the energy is dispersed across multiple foci. This dispersion does not increase with zaxis steering. The acoustic lens correction restores a single focus in every case. The surface of the focal spot increases slightly with steering.

Figure 8 quantitatively evaluates the impact of $\mathrm{z}$-axis steering on four significant parameters: the transverse and longitudinal errors, the maximum acoustic intensity, and the surface of the $-3 \mathrm{~dB}$ area. Without correction, these indicators remain constant and do not follow a monotonic trend when the steering depth increases. With correction, the longitudinal and transverse errors remain below $2 \mathrm{~mm}$ and $1 \mathrm{~mm}$ respectively, 

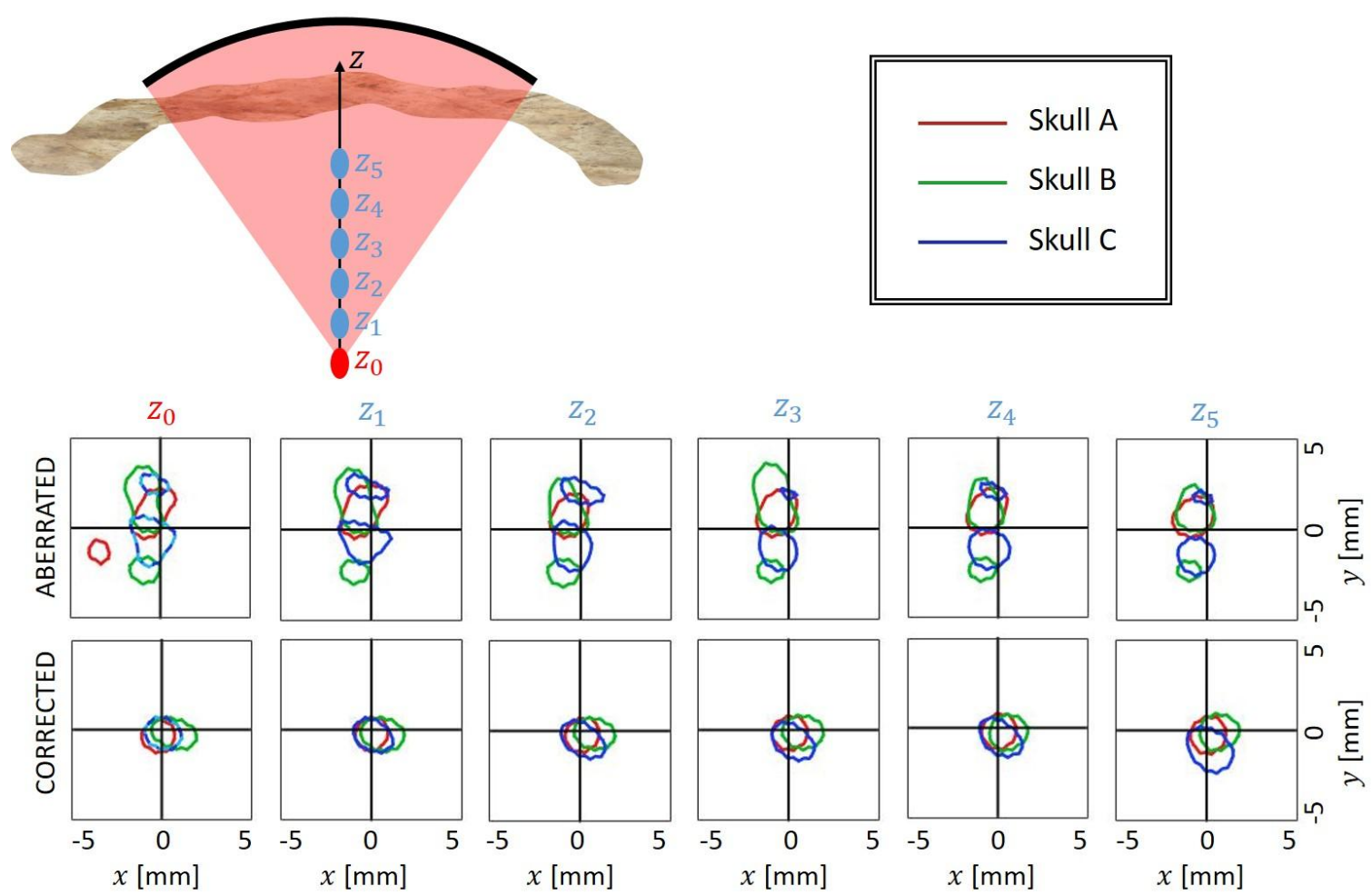

for every steering position along the $\mathrm{z}$ axis. Compared to the reference (steering-less position at $\mathrm{x}_{0}$ ), the acoustic intensity decreases by $37 \%$ experimentally (and by $60 \%$ numerically) for a $10 \mathrm{~mm}$ steering along the $\mathrm{z}$ axis. The $-3 \mathrm{~dB}$ surface does not significantly increase with steering; it varies from $2.94 \mathrm{~mm}^{2}$ (no steering) to $3.72 \mathrm{~mm}^{2}$ (10mm steering) in the experimental case, and from $1.97 \mathrm{~mm}^{2}$ to $2.77 \mathrm{~m}^{2}$ in the numerical case.

\section{DISCUSSION}

To put them in perspective, the positioning errors relatively to the target should be compared to the focal spot dimensions through its Full Width at Half Maximum (FWHM) for freespace propagation; these amount to $11 \mathrm{~mm}$ in the longitudinal direction and to $1.5 \mathrm{~mm}$ in the transverse direction respectively. In this study, without steering, the average error is $1.69 \mathrm{~mm}$ in the longitudinal direction and $0.51 \mathrm{~mm}$ in the transverse direction. The positioning errors thus correspond to $15 \%$ and $34 \%$ of the FWHM values respectively.

This study performed at $914 \mathrm{kHz}$ shows that the energy deposition significantly decreases with mechanical steering ($50 \%$ for a $10 \mathrm{~mm}$ steering) in the longitudinal direction as well as in the transverse direction. For sake of comparison, electronic steering with a transcranial multi-elements array at a similar frequency $(1 \mathrm{MHz})$ showed a $50 \%$ in energy deposition for a $20 \mathrm{~mm}$ steering in the transverse

direction[60]. As a consequence, trying to reach identical steering range for high-intensity treatments would in fine require to double the acoustic power to obtain equivalent energy levels at the focus which would mean that the patient's skull would also absorb twice as much energy. Nevertheless, compensating such steering loss would be less of an issue for low-power therapies such as BBB opening or neuromodulation.

Moreover, the steering capabilities needed for clinical translation differ with the indication. In the case of ultrasonic surgery of essential tremors the target is the ventral intermediate (VIM) nucleus. Unfortunately, the VIM is not visible on current MR images acquired at $1.5 \mathrm{~T}$ or $3 \mathrm{~T}$ and the neurosurgeon estimates the position of the VIM relatively to the anterior-posterior commissural line [10]. From this initial target, low-power sonications are first performed. Based on the physiological reactions of the patient, the neurosurgeon adjusts the position of the focus in order to pinpoint the VIM nucleus. These intraoperative adjustments require millimeter displacements of the focus around the initial target. The steering capabilities presented in this study thus appear in line with the clinical need for essential tremor treatment. The steering capability demonstrated here could also be applied to track a moving target through an aberrating medium by compensating in real-time the respiratory or heart motion.

In another application and for the first time, Coluccia et al. 

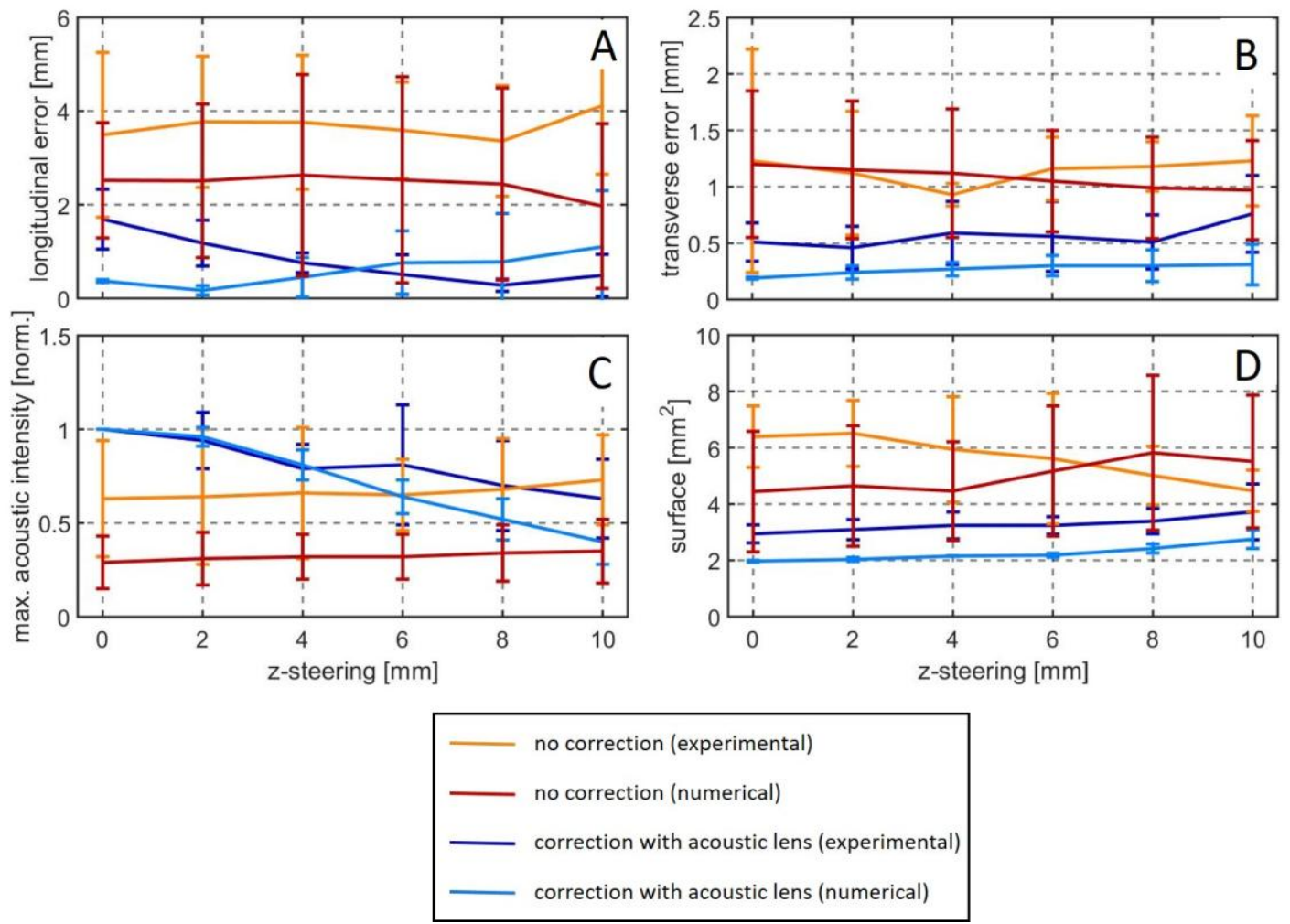

have performed in 2014 the partial ablation by focused ultrasound of a glioblastoma multiform[61]. In that specific case, the tumor volume is relatively small (equals to $6.5 \mathrm{~cm}^{3}$ ). In such small tumor cases, our steering capabilities are thus also sufficient. However, a retrospective study shows that on a sample of 135 patients suffering from glioblastoma multiform, the average tumor volume before resection could reach $34 \mathrm{~cm}^{3}$ [62], indicating tumors of width exceeding $3.5 \mathrm{~cm}$. To treat those tumors or brain metastasis, BBB opening by focused ultrasound is promising. However, the $10 \mathrm{~mm}$ displacements of the focus obtained in this study are insufficient if one hopes to achieve focusing by steering on a volume large enough to perform a large opening of the BBB. In that case, a specific lens should be designed to target a wider volume at lower intensity.

For the purpose of this study, we used the exact same acoustic lenses used in Maimbourg et al.[53]. It is worth mentioning that efficiency of the lenses was not affected after a 12-months storage and manual repositioning on the surface of the transducer. This tends to demonstrate that using the same acoustic lens for repeated treatments of the same region of the brain on the same patient is robust and should be feasible.

It is worth mentioning here that the transducer used in this proof of concept paper has a low f-number $(\mathrm{F} / \mathrm{D}=0.91)$ compared to current transcranial therapy transducers for which F/D is ranging between 0.7[56] and 0.5[63-65]. Further work needs to be done with a larger aperture transducer in order to assess the potential of the lens-based correction for transcranial thermal ablation with an antenna gain similar to current clinical transcranial ultrasonic neurosurgery devices.

The lens-based correction approach is promising for a wide range of applications. We presented here its steering capabilities for transcranial focusing. The concept was applied recently to improve the focusing through cancellous bone[66], and could be used to improve cancellous bone characterization using transmission and/or backscatter measurements[67, 68].

\section{CONCLUSION}

By contrast with a multi elements array, for which the phase profile is reconfigurable in real-time, a single-element covered with an acoustic lens is target-specific. Nevertheless, we demonstrated here that moderate steering is achievable while keeping a satisfactory quality of focusing. This study, conducted on three human skull samples, confirms that mechanical tilting of the transducer with the lens restores a single focus at $914 \mathrm{kHz}$ for a steering up to $11 \mathrm{~mm}$ in the transverse direction, and $10 \mathrm{~mm}$ in the longitudinal direction, around the focal region. This result is a step forward to using the lens-based technology in clinical applications.

\section{REFERENCES}


1. Tay, K.J., et al., Focal Therapy for Prostate Cancer with In-Bore MR-guided Focused Ultrasound: Two-Year Follow-up of a Phase I Trial-Complications and Functional Outcomes. Radiology, 2017. 285(2): p. 620-628.

2. Jones, T.A., et al., High Intensity Focused Ultrasound for RadioRecurrent Prostate Cancer: A North American Clinical Trial. The Journal of urology, 2017.

3. Farr, N., et al., Hyperthermia-enhanced targeted drug delivery using magnetic resonance-guided focussed ultrasound: a preclinical study in a genetic model of pancreatic cancer. International Journal of Hyperthermia, 2017: p. 1-8.

4. Maeda, M., et al., Sonodynamic Therapy Based on Combined Use of Low Dose Administration of Epirubicin-Incorporating Drug Delivery System and Focused Ultrasound. Ultrasound in Medicine and Biology, 2017. 43(10): p. 2295-2301.

5. $\mathrm{Gu}, \mathrm{J}$. , et al., Magnetic resonance guided focused ultrasound surgery for pain palliation of bone metastases: early experience of clinical application in China. Zhonghua yi xue za zhi, 2015. 95(41): p. 3328-3332.

6. Yeo, S.Y., et al., Bone metastasis treatment using magnetic resonance-guided high intensity focused ultrasound. Bone, 2015. 81: p. 513-523.

7. Ellens, N. and K. Hynynen, Simulation study of the effects of near-and far-field heating during focused ultrasound uterine fibroid ablation using an electronically focused phased array: A theoretical analysis of patient safety. Medical physics, 2014. 41(7).

8. Zhao, W.-P., et al., Feasibility of ultrasound-guided high intensity focused ultrasound ablating uterine fibroids with hyperintense on T2-weighted MR imaging. European journal of radiology, 2013. 82(1): p. e43-e49.

9. Okada, A., et al., Non-invasive magnetic resonance-guided focused ultrasound treatment of uterine fibroids in a large Japanese population: impact of the learning curve on patient outcome. Ultrasound in Obstetrics \& Gynecology, 2009. 34(5): p. 579-583.

10. Elias, W.J., et al., A Pilot Study of Focused Ultrasound Thalamotomy for Essential Tremor. New England Journal of Medicine, 2013. 369(7): p. 640-648.

11. Schlesinger, D., et al., Equivalence of cell survival data for radiation dose and thermal dose in ablative treatments: analysis applied to essential tremor thalamotomy by focused ultrasound and gamma knife. International Journal of Hyperthermia, 2017. 33(4): p. 401-410.

12. Hughes, A., et al., A numerical study on the oblique focus in MRguided transcranial focused ultrasound. Physics in medicine and biology, 2016. 61(22): p. 8025.

13. Pinton, G., et al., Attenuation, scattering, and absorption of ultrasound in the skull bone. Medical physics, 2012. 39(1): p. 299307.

14. Fry, F.J. and J.E. Barger, Acoustical properties of the human skull. The Journal of the Acoustical Society of America, 1978. 63(5): p. 1576-1590.

15. Tanter, M., J.-L. Thomas, and M. Fink, Focusing and steering through absorbing and aberrating layers: application to ultrasonic propagation through the skull. The Journal of the Acoustical Society of America, 1998. 103(5): p. 2403-2410.

16. Tanter, M., J.-L. Thomas, and M. Fink. Focusing through skull with time reversal mirrors. Application to hyperthermia. in Ultrasonics Symposium, 1996. Proceedings., 1996 IEEE. 1996. IEEE.

17. Sun, J. and K. Hynynen, Focusing of therapeutic ultrasound through a human skull: a numerical study. J Acoust Soc Am, 1998. 104(3 Pt 1): p. 1705-15.

18. Hynynen, K. and F.A. Jolesz, Demonstration of potential noninvasive ultrasound brain therapy through an intact skull. Ultrasound Med Biol, 1998. 24(2): p. 275-83.

19. Cohen, Z.R., et al., Magnetic resonance imaging-guided focused ultrasound for thermal ablation in the brain: a feasibility study in a swine model. Neurosurgery, 2007. 60(4): p. 593-600; discussion 600.

20. Pernot, M., et al., In vivo transcranial brain surgery with an ultrasonic time reversal mirror. J Neurosurg, 2007. 106(6): p. 1061-6.

21. Hynynen, K., et al., Pre-clinical testing of a phased array ultrasound system for MRI-guided noninvasive surgery of the brain - a primate study. European journal of radiology, 2006. 59(2): p. 149-156

22. Chauvet, D., et al., Targeting accuracy of transcranial magnetic resonance-guided high-intensity focused ultrasound brain therapy: a fresh cadaver model. Journal of Neurosurgery, 2013. 118(5): p. 1046-1052.

23. Eames, M.D., et al., Trans-cranial focused ultrasound without hair shaving: feasibility study in an ex vivo cadaver model. Journal of Therapeutic Ultrasound, 2014. 1(1): p. 1-6.

24. Marsac, L., et al., MR-guided adaptive focusing of therapeutic ultrasound beams in the human head. Medical Physics, 2012. 39: p. 1141.

25. Marquet, F., et al., Real-time, transcranial monitoring of safe blood-brain barrier opening in non-human primates. PloS one, 2014. 9(2): p. e84310.

26. McDannold, N., et al., Temporary disruption of the blood-brain barrier by use of ultrasound and microbubbles: safety and efficacy evaluation in rhesus macaques. Cancer research, 2012. 72(14): p. 3652-3663

27. Hynynen, K., et al., Noninvasive MR imaging-guided focal opening of the blood-brain barrier in rabbits. Radiology, 2001. 220(3): p. 640-6.

28. Liu, H.L., et al., Hemorrhage detection during focused-ultrasound induced blood-brain-barrier opening by using susceptibilityweighted magnetic resonance imaging. Ultrasound Med Biol, 2008. 34(4): p. 598-606.

29. Magnin, R., et al., Magnetic resonance-guided motorized transcranial ultrasound system for blood-brain barrier permeabilization along arbitrary trajectories in rodents. Journal of therapeutic ultrasound, 2015. 3(1): p. 22.

30. Marquet, F., et al., Non-invasive ultrasonic surgery of the brain in non-human primates. The Journal of the Acoustical Society of America, 2013. 134(2): p. 1632-1639.

31. Deffieux, T., et al., Low-intensity focused ultrasound modulates monkey visuomotor behavior. Current Biology, 2013. 23(23): p. 2430-2433.

32. King, R.L., et al., Effective Parameters for Ultrasound-Induced In Vivo Neurostimulation. Ultrasound in Medicine \& Biology, 2013. 39(2): p. 312-331.

33. Tufail, Y., et al., Ultrasonic neuromodulation by brain stimulation with transcranial ultrasound. Nat. Protocols, 2011. 6(9): p. 14531470 .

34. Yoo, S.-S., et al., Focused ultrasound modulates region-specific brain activity. NeuroImage, 2011. 56(3): p. 1267-1275.

35. Younan, Y., et al., Influence of the pressure field distribution in transcranial ultrasonic neurostimulation. Medical physics, 2013. 40(8): p. 082902.

36. Legon, W., et al., Transcranial focused ultrasound modulates the activity of primary somatosensory cortex in humans. Nature neuroscience, 2014. 17(2): p. 322-329.

37. Wattiez, N., et al., Transcranial ultrasonic stimulation modulates single-neuron discharge in macaques performing an antisaccade task. Brain Stimulation: Basic, Translational, and Clinical Research in Neuromodulation, 2017. 10(6): p. 1024-1031.

38. Martin, E., et al., High-intensity focused ultrasound for noninvasive functional neurosurgery. Ann Neurol, 2009. 66(6): p. 858-61.

39. Lipsman, N., et al., MR-guided focused ultrasound thalamotomy for essential tremor: a proof-of-concept study. The Lancet Neurology, 2013. 12(5): p. 462-468.

40. Chang, W.S., et al., Unilateral magnetic resonance guided focused ultrasound thalamotomy for essential tremor: practices and clinicoradiological outcomes. Journal of Neurology, Neurosurgery \& Psychiatry, 2014: p. jnnp-2014-307642.

41. Clement, G.T., et al., A hemisphere array for non-invasive ultrasound brain therapy and surgery. Phys Med Biol, 2000. 45(12): p. 3707-19.

42. Pernot, M., et al., High power transcranial beam steering for ultrasonic brain therapy. Phys Med Biol, 2003. 48(16): p. 257789.

43. Jeanmonod, D., et al., Transcranial magnetic resonance imagingguided focused ultrasound: noninvasive central lateral thalamotomy for chronic neuropathic pain. Neurosurgical Focus, 2012. 32(1): p. 1-11. 
44. Song, J. and K. Hynynen, Feasibility of using lateral mode coupling method for a large scale ultrasound phased array for noninvasive transcranial therapy. IEEE Trans Biomed Eng, 2010. 57(1): p. 124-33.

45. Lele, P., An annular-focus ultrasonic lens for production of uniform hyperthermia in cancer therapy. Ultrasound in Medicine \& Biology, 1981. 7(2): p. 191-193.

46. Umemura, S., C. Cain, and K. Katakura. Acoustic field synthesis by sector-vortex array for tumor treatment. in IEEE 1986 Ultrasonics Symposium. 1986. IEEE.

47. Cain, C.A. and S.-I. Umemura, Concentric-ring and sector-vortex phased-array applicators for ultrasound hyperthermia. IEEE Transactions on Microwave Theory and Techniques, 1986. 34(5): p. 542-551.

48. Lalonde, R., A. Worthington, and J. Hunt. Hyperthermia: Field conjugate acoustic lenses for deep heating. in Engineering in Medicine and Biology Society, 1990., Proceedings of the Twelfth Annual International Conference of the IEEE. 1990. IEEE.

49. Takayama, N. and T. Itoh. Investigation of ultrasonic heating with a non-axial symmetric acoustic lens. in Proc. 5th Int. Symp. Hyperthermic Oncology. 1989.

50. Fjield, T. and K. Hynynen, The combined concentric-ring and sector-vortex phased array for MRI guided ultrasound surgery. IEEE transactions on ultrasonics, ferroelectrics, and frequency control, 1997. 44(5): p. 1157-1167.

51. Brown, M., et al., Generating arbitrary ultrasound fields with tailored optoacoustic surface profiles. Applied Physics Letters, 2017. 110(9): p. 094102.

52. Fjield, T., C.E. Silcox, and K. Hynynen, Low-profile lenses for ultrasound surgery. Phys Med Biol, 1999. 44(7): p. 1803-13.

53. Maimbourg, G., et al., 3D-printed adaptive acoustic lens as a disruptive technology for transcranial ultrasound therapy using single-element transducers. Physics in Medicine \& Biology, 2018. 63(2): p. 025026.

54. Jones, M.W., et al., Sub-transducer element spatial resolution ultrasound wavefront modification using passive twin-layer compensators. Applied Acoustics, 2018. 131: p. 129-133.

55. McKechnie, T.S., Atmospheric Isoplanatic Angle. in General theory of light propagation and imaging through the atmosphere. 2016, Springer.

56. Marsac, L., et al., Ex vivo optimisation of a heterogeneous speed of sound model of the human skull for non-invasive transcranial focused ultrasound at $1 \mathrm{MHz}$. International Journal of Hyperthermia, 2017: p. 1-11.

57. Cox, B.T., et al., $k$-space propagation models for acoustically heterogeneous media: Application to biomedical photoacoustics. The Journal of the Acoustical Society of America, 2007. 121(6): p. 3453-3464.

58. Duck, F.A., Physical properties of tissues: a comprehensive reference book. 1990: Academic Press.

59. Barriere, C. and D. Royer, Optical measurement of large transient mechanical displacements. Applied Physics Letters, 2001. 79(6): p. 878-880.

60. Gateau, J., et al., Transcranial ultrasonic therapy based on time reversal of acoustically induced cavitation bubble signature. IEEE Trans Biomed Eng, 2010. 57(1): p. 134-44.

61. Coluccia, D., et al., First noninvasive thermal ablation of a brain tumor with MR-guided focusedultrasound. Journal of therapeutic ultrasound, 2014. 2(1): p. 17.

62. Kuhnt, D., et al., Correlation of the extent of tumor volume resection and patient survival in surgery of glioblastoma multiforme with high-field intraoperative MRI guidance. Neurooncology, 2011. 13(12): p. 1339-1348.

63. Eames, M.D., et al., Head phantoms for transcranial focused ultrasound. Medical physics, 2015. 42(4): p. 1518-1527.

64. Arvanitis, C.D., G.T. Clement, and N. McDannold, Transcranial assessment and visualization of acoustic cavitation: modeling and experimental validation. Medical Imaging, IEEE Transactions on, 2015. 34(6): p. 1270-1281.

65. Ondo, W., et al., An international, randomized, sham controlled trial of focused ultrasound thalamotomy for essential tremor. Movement Disorders, 2016. 31: p. S49.

66. replica models. Journal of Tissue Engineering, 2018. 9: p. 2041731418766418.

67. Chaffa1, S., et al., Ultrasonic characterization of human cancellous bone using transmission and backscatter measurements: relationships to density and microstructure. Bone, 2002. 30(1): p. 229-237.

68. Wear, K.A., et al., Relationships among ultrasonic and mechanical properties of cancellous bone in human calcaneus in vitro. Bone, 2017. 103: p. 93-101. 